\title{
CONSIDERACIONES EN TORNO A LOS MÉTODOS DE ENSEÑANZA DE LENGUAS EXTRAJERAS Y A LAS TEORÍAS DE APRENDIZAJE
}

\author{
Giorgia Marangon
}

\begin{abstract}
RESUMEN
Este artículo se centra en la evolución histórica de los métodos de enseñanza de las lenguas extranjeras, desde el latín como lengua culta, a las actuales lenguas modernas. La idea del método idóneo ha quedado atrás por utópica e inalcanzable, no existe la perfección metodológica, sino un saber hacer y adaptación constante de métodos, teorías, enfoques y estilos de enseñanzaaprendizaje. Este recorrido en torno a los métodos de enseñanza de lenguas extranjeras y las teorías de aprendizaje tiene como objetivo enfocar la atención del lector sobre los pros y los contras de dichos métodos y sobre la repercusión que tuvieron a lo largo de la historia.

Palabras clave: Método, enseñanza, lenguaje, aprendizaje, lenguas extranjeras.
\end{abstract}

\begin{abstract}
This article focuses on the historical development of methods of teaching foreign languages, from Latin as the language of scholars to current modern languages. Considered utopian and unattainable, the idea of the perfect method was left behind as there is no methodological perfection, but rather a know-how and constant adaptation of methods, theories, approaches and styles of teaching/ learning. This article highlights the different methods of foreign language teaching and learning theories with the purpose of demonstrating to the reader the pros and cons, as well as their historical impact.
\end{abstract}

Key words: Methodology, teaching, language, learning, foreing languages.

\section{Introducción}

Los profundos cambios sociales y políticos de finales del siglo XVIII y principios del XIX que tienen lugar en Europa -entre ellos la Revolución Industrial, y con ella el incremento de relaciones comerciales entre distintos países y culturas- hacen que surjan las primeras propuestas

Dra. Giorgia Marangon. Profesora. Departamento de Traducción e Interpretación, Lenguas Romances, Estudios Semíticos y Documentación. Universidad de Córdoba.

Correo electrónico: 1r1marmg@uco.es

Recepción: 16- 05- 2012

Aceptación: 27- 09- 2012 
para la enseñanza de lenguas extranjeras. Así, en los planes de estudio de las universidades de Gran Bretaña, Francia y Alemania, la docencia de lenguas extranjeras comienza, en el siglo XIX, a acompañar la de lenguas clásicas como el latín o el griego.

La desvinculación de la enseñanza del ámbito estrictamente eclesiástico, defendida por autores como J. Locke1, se opone al sentir de la burguesía, que estimaba que estas "nuevas lenguas" tenían una dificultad mucho menor que la latina o la griega. Por esta razón, cuando se inicia la enseñanza de lenguas extranjeras se hace siguiendo la misma metodología que la aplicada a las lenguas clásicas, es decir, mediante el conocimiento de las reglas gramaticales y morfosintácticas.

La práctica, como empleo de todas estas normas dentro de una conversación, ocupaba un lugar secundario. No obstante, este autor, haciendo referencia a la enseñanza del francés como lengua extranjera, aboga por el ejercicio oral y los diálogos como método de enseñanza; es más, antepone el aprendizaje de esta lengua a la del latín:

\begin{abstract}
As soon as he can speak English 'tis time for him to learn some other Language: This no body doubts of, when French ist proposed. And de Reason is, because People ar accustomed to the right way of teaching that Language, which is by talking into Children in constant Conversation, and not by Grammatical Rules. The Latin Tongue would easily be taught the fame way if his Tutor, being constantly with him, would talk nothing else to him, and make him answer still in the fame Language. But because French is a living Language, and to be used more in speaking, that should be first learn'd, that the yet pliant Organs of Speech might be accustomed to a due formation for those Sounds, and he get the habit of pronouncing French well, which is the harder to be done the longer it is delay'd.

When he can speak and read French well, which in this Method is ufually in a Year or two, he should proceed to Latin, which 'tis a wonder Parents, when they have had the experiment in French, should not think ought to be learn'd the same way, by talking and reading [...]. (Locke 1693: 192)
\end{abstract}

Pero, ¿qué significa el lema "método"? Método significa el camino para llegar a un resultado determinado. Así, el método que utilicemos nos facilitará el camino, nos pondrá más dificultades, o nos desviará definitivamente de nuestro destino inicial. El método es parte fundamental en cualquier trabajo que se precie de ser llamado científico, y asimismo de cualquier actividad educativa. Con una buena aplicación del método o métodos más correctos, se conseguirán resultados educativos serios, rigurosos y formales. El Longman Dictionary of Language Teaching and Applied Linguistics, en su edición de 1997, define el método como una forma de enseñar una lengua que se basa en principios y procedimientos sistematizados que a su vez representan la concepción de cómo la lengua es enseñada y aprendida. Según el Longman Dictionary, los métodos difieren unos de otros en su concepción sobre la naturaleza del lenguaje y su aprendizaje, en los propósitos y objetivos de enseñanza, en el tipo de programa que promueve, las técnicas y procedimientos que recomienda y el papel que le asignan al profesor, a los aprendices y a los materiales instructivos.

En la enseñanza de lenguas se distinguen entre métodos generales y específicos, tradicionales y contemporáneos; más sin hacer válidas estas distinciones -entre otras razones por vagas e imprecisas-, la literatura ha documentado un sinnúmero de métodos, métodos diseñados para enseñar la lengua materna (método auditivo, alfabético o fónico, linguiístico, de la oración, de la palabra, de la vista, etc.) y aquellos diseñados o utilizados particularmente para enseñar lenguas extranjeras o segundas lenguas (método directo instrumental, audiovisual, etc.), que en ocasiones se confunden. Dada esta situación, consideramos más práctico analizar tan solo aquellos que han tenido una larga historia de influencias en la enseñanza de lenguas extranjeras y que aún mantienen vivas sus raíces en los métodos contemporáneos. 


\section{Evolución histórica de los métodos de enseñanza de las lenguas extranjeras}

\subsection{Métodos directos}

A finales del siglo XIX aparecen los primeros métodos directos ${ }^{2}$, que propugnan una "inmersión" del alumno en la lengua objeto de estudio. Para ello, en ningún caso se emplea la lengua materna, así como tampoco se ofrecen explicaciones de las reglas gramaticales, morfológicas o sintácticas. Surge como reacción al método gramática-traducción que es el más viejo y ortodoxo de los métodos de enseñanza de lenguas que reinó durante el siglo XVIII y parte del XIX, y debe su origen a las escuelas de latín, donde fue ampliamente usado para enseñar las lenguas "clásicas" (latín y griego), aunque más tarde se utilizó también para enseñar algunas lenguas modernas (francés, alemán e inglés). La traducción de una lengua a la otra servía como técnica principal para explicar las nuevas palabras, las formas y estructuras gramaticales, y como vía óptima de llegar al dominio de la lengua en general. Los métodos directos implicaban, pues, que el docente debía valerse de medios tales como fotografías, imágenes, objetos, etc. a la hora de explicar el significado de los distintos vocablos. En lo concerniente a la morfosintaxis, se recurría a la repetición de fórmulas predeterminadas. Sin embargo, tampoco estos métodos serán considerados adecuados para la enseñanza de lenguas extranjeras. Los principales escollos que presentan son los siguientes:

a. Hay una gran influencia de la lengua materna sobre la lengua extranjera en proceso de asimilación, lo cual impide, en cierta forma, que esta segunda lengua pueda asimilarse con la misma agilidad que la materna.

b. Al aprender la lengua materna, además de los elementos lingüísticos, existen otros de carácter extralingüístico, como pueden ser determinados conceptos, la interpretación de la entonación del hablante o del entorno, etc. Esto no es posible en el caso del aprendizaje de una segunda lengua, a menos que dicho aprendizaje se haga de forma prolongada y continua y en el país de origen, pues la necesidad de comunicación para cubrir necesidades básicas, tales como dormir, comer, encontrar un camino, pedir asistencia médica, etc., hacen que el discente se encuentre en la misma situación que cuando era niño y necesitaba transmitir sus deseos de hambre, sed, sueño, malestar, etc.

c. El aprendizaje de la lengua materna no puede ser equiparable al aprendizaje en edad adolescente o adulta: en el primer caso el aprendizaje está motivado por la necesidad vital de comunicarnos, esto favorece la rápida asimilación de toda la información lingüística, aunque sea de manera inconsciente; por el contrario, en el aprendizaje de lenguas en edad adulta existen factores tales como la falta de atención, el cansancio, la distracción, etc. que impiden que la asimiliación tenga las características anteriormente mencionadas.

d. El empleo exclusivo de la "inmersión" lingüística, sin recurrir en ningún momento a una explicación léxica, morfológica o sintáctica puede provocar confusiones y falsas identidades, especialmente si la lengua materna del discente tiene una raíz común a la segunda lengua objeto de estudio -como es, por ejemplo, el caso del español y el italiano, del español y el francés-.

No obstante, y a pesar de que, como ya hemos apuntado, enseguida se desechó la utilidad innovadora de estos métodos, a lo largo de todo el pasado siglo siguieron empleándose, pero siempre en combinación con cualquier otra metodología. 


\subsubsection{Métodos instrumentales}

Tras la Segunda Guerra Mundial comienza a emplearse el denominado método instrumental o fundamentado, debido principalmente a la necesidad urgente de aprender idiomas tales como el inglés, el francés, el alemán o el italiano, dados los acontecimientos bélicos que acababan de conmocionar al mundo ${ }^{3}$. Es esta una enseñanza instrumental de las lenguas extranjeras en la que se recurre a criterios puramente científicos y que tiene como características principales las siguientes:

a. Se trata de un aprendizaje muy funcional, por lo que se selecciona el léxico y la gramática necesarios para desempeñar la actividad para la cual es necesario el idioma (transacciones económicas y comerciales, operaciones jurídico-militares, etc.)

b. Se transmiten aspectos gramaticales y léxicos sin que existan consideraciones de tipo antropológico, sociológico o ideológico referentes al país de origen de la lengua objeto de estudio.

c. La planificación de las materias que van a ser impartidas es exacta; por ello, la estructuración de los contenidos léxicos y gramaticales es muy pormenorizada.

d. Esta metodología recurre a los denominados métodos audiovisuales, lo cual permite enseñar la lengua a un número muy elevado de alumnos. Esto posibilita, enseñar a colectivos amplios, como pueden ser, por ejemplo, los empleados de empresas multinacionales.

\subsubsection{Métodos audio-orales}

Este método, empero, exigía una infraestructura realmente costosa, por lo que a finales de los años setenta, debido fundamentalmente a las crisis económicas, comienzan a caer en desuso. En su lugar se trabaja con las denominadas dinámicas audio-orales, que logran conciliar la metodología clásica y la directa, sin olvidar la fundamentación lingüística de las instrumentales o fundamentadas. Estas dinámicas se basan en la comunicación oral y en el análisis inmanente de la lengua, al tiempo que propugnan el aprendizaje de la gramática y de las reglas morfosintácticas. En esta nueva metodología de enseñanza-aprendizaje cobran especial relevancia una serie de estructuras gramaticales determinadas - patterns-, a partir de las cuales el alumno puede desarrollar otras estructuras gramaticales similares. Así, en el caso, por ejemplo, del aprendizaje del italiano como lengua extranjera, a partir de la flexión en presente de indicativo de verbos como "parlare, vedere, partire", el estudiante puede conjugar otros como "cantare, credere, sentire" siempre que respeten las mismas pautas, en este caso el hecho de ser verbos regulares.

En lo concerniente a la sintaxis, partiendo de oraciones como "Voglio che tu venga" (Quiero que tú vengas), el estudiante puede formar otras similares, del tipo "Voglio che tu venga con me", siguiendo siempre la estructura: sujeto + verbo volere + pronombre en acusativo + subjuntivo. Esto permite, al mismo tiempo, establecer una comparación de la segunda lengua con la lengua materna. No obstante, también la metodología audio-oral contiene inconvenientes a la hora de ser aplicada:

a. La práctica del pattern hace que la unidad didáctica, y con ello las clases de lengua, se conviertan en algo monótono, pues tan sólo se basan en la repetición de estructuras por 
parte del estudiante. Esto hace que la motivación del alumno vaya disminuyendo de forma progresiva, especialmente en el caso de aquellos que ya poseen conocimientos de la lengua objeto de estudio.

b. El establecimiento de los patterns por parte del docente veta la posibilidad de reflexión por parte del alumnado, que desempeña un papel fundamentalmente pasivo al limitarse a aplicar las mismas estructuras una y otra vez: el estudiante no se plantea por qué ha de emplear una determinada estructura sintáctica o una categoría morfológica concreta y no otras, puesto que previamente, ya se le ha facilitado la labor de pensar cuáles de estas estructuras son las más adecuadas.

c. Del mismo modo, tampoco se facilita la reflexión sobre el significado y la utilidad de los términos empleados o de los elementos oracionales que poco a poco se asimilaban a partir de una estructura básica.

\subsubsection{Métodos audiovisuales}

Junto a este método también se emplean, con posterioridad a la Segunda Guerra Mundial, los métodos audiovisuales, que cobran una especial relevancia, pues dejan de ser una herramienta auxiliar para el aprendizaje de la L2, y se convierten en parte fundamental del método ${ }^{4}$. El objetivo de estos métodos es hacer llegar las diferentes lenguas al gran número de inmigrantes existente en la mayor parte de los países de Europa tras la Segunda Guerra Mundial. Ello justifica que la pragmática del lenguaje y la selección del léxico adecuado a las necesidades fundamentales de los alumnos sean elementos fundamentales para su aplicación.

Las dos fases fundamentales que intervienen en estos métodos son las siguientes:

a. Fase de recepción. Dentro de esta fase cabe distinguir tres momentos relevantes, que son los de presentación de los contenidos lingüísticos, explicación de dichos contenidos y repetición de los mismos.

b. Fase de producción. Esta fase consiste en la explicación sobre la imagen, la explotación gramatical de dicha imagen y la aplicación de los contenidos explicados a otras situaciones similares.

Los detractores de este tipo de métodos argumentan que el estudiante queda condenado a una inactividad total, amén de la inexistencia de interacción y cooperación entre los participantes de la unidad didáctica. Además, contempla una falta de motivación y de estímulo en el alumno que recibe la enseñanza de una lengua extranjera mediante estos métodos.

En la actualidad, el objetivo fundamental de la enseñanza de toda lengua extranjera es que el alumno alcance una competencia comunicativa, esto es, la capacidad de comunicación suficiente como para valerse en la lengua que está aprendiendo. Siguiendo este objetivo fundamental surge la denominada pragmadidáctica, que se basa en la función social y cultural que toda lengua tiene. Enunciada por Austin y Searle, la pragmadidáctica concibe al hablante como homo sociabilis, y no emplea un método determinado, sino que para conseguir su objetivo -la competencia comunicativa del estudiante en la lengua extranjera cursada- no duda, en caso de ser necesario, en emplear varios de los métodos anteriormente expuestos. En este sentido, cabe señalar lo expuesto por Stephan Schlickau (2000), quien defiende el uso de vídeos y de la videoconferencia en el aprendizaje de una lengua extranjera. Según Richard Vaughan (2008), experto en los pormenores de la enseñanza del inglés con alumnado adulto, 
la aplicación de estos métodos audiovisuales para la enseñanza y su empleo posibilita al alumnado una situación comunicativa auténtica, y dada esa autenticidad que muestra el diálogo, se produce una mayor motivación en el estudiante. Ahora bien, añade que hay que saberlos usar para que el alumno no se desmotive. Son, por tanto, características positivas para la enseñanza:

a. La presentación de conversaciones reales llevadas a cabo por hablantes nativos, lo que reduce la artificiosidad del lenguaje.

b. La videoconferencia, a su vez, posibilita una forma directa de comunicación que es posible sin necesidad de realizar un viaje al país de origen.

c. El estudiante puede discutir, debatir, preguntar o manifestar su opinión sobre cuestiones acuales.

Un caso parecido es el de la utilización en el aula de aprendizaje de lenguas extranjeras de películas en versión original. Así, autores como Sass, afirman que vídeos, que han tenido y tienen proyección internacional, pueden resultar beneficiosos para enseñar una segunda lengua extranjera, puesto que, afirma, el estudiante se identifica no sólo con datos y hechos, sino también con sentimientos, y todo ello conforma una fusión ideal para el aprendizaje de la lengua:

Dies ist die große Schatz, den Filme in den Unterricht transportieren, denn sie erzählen von Menschen und sie erzählen Geschichten. Wenn diese Geschichten mit den Erfahrungen der Lernenden korrespondieren oder kollidieren, ist dies ein wahrer Fundus für authentische Sprechanlässe: Die Lernenden sprechen über Geschichten und Gefühle oder besser gesagt: über ihre Geschichten und ihre Gefühle. (Sass 2007)

Por tanto, el empleo de películas en la clase de $\mathrm{L} 2^{5}$ resulta útil si:

a. Narran historias interesantes para el alumnado.

b. Provocan la curiosidad del espectador en asuntos relacionados con el país destinatario.

c. Provocan una inmersión en un mundo distinto al materno, y con ello, un nuevo punto de vista en el espectador.

d. Muestran algo inesperado.

e. Incluyen algo nuevo, interesante para ser comunicado, para reflexionar sobre él, o para debatirlo.

El uso de películas puede contribuir a aumentar la competenia comunicativa del alumno. Para ello, según la autora, el docente puede llevar a cabo distintos tipos de ejercicios:

a. Ejercicios previos a la proyección:

i. El docente puede ofrecer a los estudiantes un vocabulario nuevo sobre la película, y así, hablar sobre las relaciones personales que se entablan en ella, sobre el desarrollo de la acción, sobre la caracterización de los personajes y las situaciones, etc.

ii. Además, se puede hablar sobre el ámbito cultural, buscando similitudes y divergencias entre la cultura de la L1 de los alumnos y de la L2 estudiada.

iii. Se puede hablar, desde un punto de vista descriptivo, del vestuario, el peinado, los gestos, etc.

b. Ejercicios durante la proyección. Puesto que los argumentos y personajes que aparecen en una película suelen resultar algo complejos, no es adecuado formular preguntas sobre lo visualizado (Sass 2007). Sin embargo, un buen ejercicio sería pedir a un número determinado de estudiantes que se concentren en un determinado personaje, para describirlo tras la proyección. 
c. Ejercicios tras la proyección. Mediante las herramientas gramaticales y comunicativas adquiridas en clase hasta la proyección de la película, el alumno puede llevar a cabo dos tipos de actividades:

i. Contar la historia narrada.

ii. Dar un final alternativo a la narración.

A lo anteriormente expuesto, cabe añadir que las películas:

a. Posibilitan el conocimiento de la realidad cultural en la que la lengua extranjera aprendida está inmersa.

b. Ofrecen muchas conexiones con la actividad diaria.

c. Amplían la perspectiva intercultural.

d. Sirven para el aprendizaje de la L2 como lengua extranjera para jóvenes y adultos.

e. Pueden emplearse junto a otras tecnologías, como por ejemplo las informáticas y las multimedias.

\subsubsection{Métodos interactivos}

Recientemente, ha cobrado gran relevancia el empleo de los denominados métodos interactivos, que nacen gracias a las nuevas tecnologías. Las denominadas enseñanzas de lenguas asistidas por ordenador (Computer Aided Language Learning, CALL) han "revolucionado" la enseñanza y el aprendizaje de las segundas lenguas. Casado y García Bermejo afirman a este respecto:

Hasta hace solamente una década, el uso de ordenadores en la clase de idiomas era algo que estaba relegado a unos pocos especialistas en el tema. Sin embargo, con el desarrollo de la tecnología multimedia y la implantación cada vez más extendida de Internet, el papel de los ordenadores en la clase de lengua extranjera se ha convertido en un tema de gran importancia en el que están implicados cada vez un mayor número de profesores en todo el mundo. (2000: 70)

La aparición del ordenador como "descubrimiento" para la enseñanza de lenguas extranjeras cuenta con una historia de aproximadamente 30 años. A este respecto afirma Breindl:

[...] Die Entwicklung geht auf der Hardware-Seite von den kosten- und wartungsintensiven aber langsamen Großrechenanlagen der 60er-Jahre über die Revolution durch den Chip in den 70er-Jahren zu immer kleineren, schnelleren und kostengünstigeren Mikrocomputern „,ür jedermann“ und erreicht durch die heutigen Möglichkeiten der Telekommunikation mit dem Internet eine neue inhaltliche Dimension. (1987: 289)

Así, el docente tiene a su alcance herramientas de trabajo tan útiles como pueden ser Internet, los CD-Rom, las tarjetas de audio y sonido disponibles en los equipos informáticos, y otros recursos multimedia ${ }^{6}$. Refiriéndose a internet, Casado y García Bermejo expone:

\footnotetext{
El enorme potencial de Internet ha dado lugar a un nuevo modelo de enseñanza a distancia, basado íntegramente en la red, conocido como e-learning o aprendizaje virtual. En este paradigma la World Wide Web [...] es utilizada para distribuir los contenidos del curso, mientras que el correo electrónico, las listas de distribucion, chats, y otros medios de comunicación son usados para el intercambio de información entre alumnos o para las tutorías entre profesor y estudiantes. (2000)
}

En efecto, el acceso a la información es una de las principales ventajas de internet, tanto para el docente como para el discente: posibilita la inclusión de numeroso material, ya sea de carácter didáctico o no didáctico, que está accesible de un modo directo y rápido ${ }^{7}$. Por otra parte, 
la web es una "inagotable fuente de información a un coste muy reducido, pues generalmente esa información es ofrecida de manera gratuita, por lo que el usuario sólo tiene que asumir los gastos de conexión" (Ruipérez 1997: 13). Anotemos dos ejemplos, uno de alemán y otro de italiano. El primero es el proyecto Prim@, primer curso de alemán para hispanohablantes. Desarrollado por los profesores Germán Ruipérez, María Dolores Castrillo y José Carlos García Cabrero (Universidad de Educación a Distancia), la versión original de este método vió la luz en el Herder-Institut de la Universidad de Leipzig, bajo la dirección de Barbara Wotjak. El proyecto contiene tres niveles: Prim@1, Prim@2 y Prim@ 3, y es el método empleado para cursar alemán como lengua extranjera en las titulaciones de Filología Inglesa y Filología Hispánica, en la Diplomatura de Turismo del la UNED y en los cursos de idiomas del Centro Universitario de Idiomas a Distancia (CUID). Otros ejemplos significativos en el ámbito de la enseñanza-aprendizaje de la lengua italiana son los portales que relacionamos a continuación, siendo siempre la red el soporte y el medio que posibilita el acceso a los contenidos que ofrecen:

Tabla 1. Algunos recursos electrónicos disponibles en la red para el aprendizaje del italiano

\begin{tabular}{|c|c|}
\hline Sitios de Internet & Descripción \\
\hline http://www.italica.rai.it/ & $\begin{array}{l}\text { Se trata de una página web que ofrece, tanto un } \\
\text { amplio abanico cultural y social dentro del marco } \\
\text { lingüístico del italiano, como cursos de aprendizaje } \\
\text { dividido en un bloque gramatical, otro de material } \\
\text { de audio, otros de lecturas, vocabulario, recursos e } \\
\text { incluso un foro y un chat para practicar el idioma. }\end{array}$ \\
\hline http://www.educational.rai.it/ioparloitaliano/main.htm & Cursos, foros, biblioteca y certificaciones oficiales \\
\hline http://www.socrates-me-too.org/ & $\begin{array}{l}\text { Esta página contiene numerosos ejercicios } \\
\text { interactivos y, aunque sirva para mejorar la destreza } \\
\text { lectora o de la escritura, el recurso que consideramos } \\
\text { más importante (aunque breve) es el bloque de } \\
\text { ejercicios auditivos que el estudiante va a encontrar. }\end{array}$ \\
\hline http://www.ciid.it/lalita/ & $\begin{array}{l}\text { En esta página web el estudiante va a encontrar } \\
\text { unos grupos temáticos que presentan una grabación } \\
\text { por hablantes nativos, acompañada de un texto } \\
\text { escrito y unas actividades de opción múltiple con } \\
\text { el fin de medir el nivel de comprensión alcanzado. }\end{array}$ \\
\hline $\begin{array}{l}\text { http://parliamoitaliano.altervista.org/ } \\
\text { http://webs.racocatala.cat/llengua/it/ }\end{array}$ & $\begin{array}{l}\text { Estos portales ofrecen más de cien ejercicios de } \\
\text { listening donde participan hablantes con diferentes } \\
\text { acentos, lo cual resulta de mucha utilidad a la } \\
\text { hora de aprender el idioma. Las locuciones vienen } \\
\text { acompañadas de los textos escritos. Además } \\
\text { presenta otros apartados tales como canciones, } \\
\text { ejercicios, noticias, juegos, videos, etc. }\end{array}$ \\
\hline
\end{tabular}

El empleo de la ELAO (Enseñanza, Lenguas, Asistida por Ordenador) se inicia en la década de los sesenta del siglo pasado, y hasta la actualidad, la enseñanza asistida por ordenador ha experimentado tres etapas claramente diferenciadas:
a. Etapa behaviorista o conductista
b. Etapa comunicativa
c. Etapa integradora 
La etapa behaviorista o conductista aparece hacia los años cincuenta, y se desarrolla en la década de los sesenta y setenta. Siguiendo el modelo conductista, este modelo de ELAO se caracterizaba por presentar al alumno una serie de ejercicios gramaticales mecánicos y repetitivos. El ordenador, pues, constituía un mero tutor mecánico, de forma que los estudiantes trabajaban de forma individual y a su propio ritmo.

A finales de los años setenta se inicia la etapa comunicativa de la ELAO, de forma que las actividades llevadas a cabo por el ordenador se centraban fundamentalmente en el uso del idioma, y no tanto en las estructuras gramaticales. Con ello, se pretendía animar a los estudiantes a producir sus propias frases, siendo, pues, el objetivo principal conseguir el desarrollo de la competencia comunicativa de los discentes. Las características principales de este tipo de enseñanza-aprendizaje son, según Casado y García Bermejo:

\begin{abstract}
El CALL comunicativo estaba en consonancia con las teorías cognitivas que proponían la enseñanza como proceso de descubrimiento, expresión y desarrollo. El software utilizado por este enfoque se basaba en programas de reconstrucción de textos, que permitían a los estudiantes, trabajando solos o en grupo, reagrupar las palabras y las frases para descubrir estructuras o significados de la lengua. Lo importante, en realidad, no era lo que los estudiantes hacían con el ordenador, sino lo que hacían unos con otros en la lengua extranjera, al mismo tiempo que utilizaban el ordenador. (2000: 72)
\end{abstract}

Finalmente, la ELAO integradora se configura como un claro avance en la enseñanza asistida por ordenador, y así, a principios de los noventa, casi a las puertas del siglo XXI, el ordenador sirvió para la enseñanza de lenguas empleando el denominado enfoque por tareas o proyectos, que presenta las siguientes características:

[...] Este enfoque, que actualmente cuenta con muchos seguidores, busca muy especialmente implicar a los alumnos en el uso de la lengua dentro de situaciones reales de comunicación, desarrollando las distintas destrezas del lenguaje. Todo esto ha conducido a una nueva perspectiva que ha sido denominada «CALL integrador», que intenta precisamente poner las nuevas tecnologías al servicio del aprendizaje de lenguas de la forma más integradora posible, donde el ordenador deja de ser un elemento añadido para facilitar al alumno una serie de herramientas fundamentales para aprender a usar la lengua. (2000: 72)

Tabla 2.

\begin{tabular}{|l|l|l|l|l|}
\hline Etapa ELAO & \multicolumn{1}{|c|}{ Tecnología } & \multicolumn{1}{|c|}{$\begin{array}{c}\text { Enfoque } \\
\text { pedagógico }\end{array}$} & $\begin{array}{c}\text { Método } \\
\text { Lingüístico }\end{array}$ & \multicolumn{1}{c|}{$\begin{array}{c}\text { Rol del } \\
\text { ordenador }\end{array}$} \\
\hline $\begin{array}{l}\text { Conductista } \\
(60-80)\end{array}$ & Macro ordenadores & Conductismo & Estructuralista & Tutor \\
\hline $\begin{array}{l}\text { Comunicativa } \\
(80-90)\end{array}$ & $\begin{array}{l}\text { Ordenador personal } \\
\text { (PC) }\end{array}$ & Cognitivismo & Communicativo & $\begin{array}{l}\text { Tutor y } \\
\text { Herramienta }\end{array}$ \\
\hline $\begin{array}{l}\text { Integradora } \\
(90-\text { hoy })\end{array}$ & $\begin{array}{l}\text { Multimedia y World } \\
\text { Wide Web }(\mathrm{www})\end{array}$ & $\begin{array}{l}\text { Sociocognitivismo } \\
\text { Constructivismo }\end{array}$ & $\begin{array}{l}\text { Combinación de } \\
\text { métodos }\end{array}$ & $\begin{array}{l}\text { Tutor y } \\
\text { Herramienta }\end{array}$ \\
\hline
\end{tabular}

El hipertexto y los entornos hipermediales constituyen, pues, una auténtica "revolución" a la hora de enseñar-aprender lenguas extranjeras y específicas. Desde un punto de vista metodológico, constituye una vía de apoyo a métodos tradicionales muy efectiva, ya que la presentación del INPUT determina en buena medida la asimilación de los contenidos. Gracias al hipertexto, estos contenidos pueden ser procesados de forma diversa: con voz, con voz y texto, con voz, texto, imágenes y otros sonidos, etc. Cuantos más sistemas sensoriales se activen, mejor queda procesado en la memoria del contenido (Storch 1997: 223). Otras ventajas de los sistemas hipermediales señaladas por el mismo autor (1998: 177): 
a. Permiten tareas desde ejercicios simples a los más complejos, para entrenar la pronunciación y la destreza oral.

b. Posibilitan el contacto con personas ajenas a la clase, como por ejemplo, hablantes nativos y estudiantes y profesores de otros centros.

c. Constituyen una herramienta auxiliar que puede ayudar a una mejor comprensión de la gramática, de los textos escritos y de las audiciones (listening).

d. Permiten la búsqueda rápida de información sobre cuestiones culturales.

e. Gracias a programas como, por ejemplo, los correctores ortográficos, se reduce el número de errores en la producción escrita.

Según Casado y García Bermejo, para un buen uso de las nuevas tecnologías en la enseñanza y aprendizajes de lenguas extranjeras, se ha de

\begin{abstract}
hacer frente a esta nueva realidad, aceptando las posibilidades que ofrecen las nuevas tecnologías y sabiendo orientar su aplicación de forma positiva para evitar posibles desequilibrios generados por un uso puramente mecánico y cómodo. [...] los profesores debemos ser los primeros en aceptarlas como una herramienta cada vez más imprescindible para nuestra labor educativa, pero sin temor a que en ningún caso puedan llegar a sustituirnos. (2000: 70)
\end{abstract}

No obstante, no hemos de olvidar que sigue siendo importantísimo el papel del profesor en el aula. Se hace necesario, empero, un replanteamiento del perfil del profesor de lengua extranjera, que ahora ha de ser algo más que un simple mediador de la información y del aprendizaje. En la actualidad, el docente ha de ser la guía para la enseñanza y el aprendizaje de los contenidos. Él es quien ha de seleccionar y ofrecer al discente el material y las herramientas más adecuadas para la adquisición de los conocimientos linguiísticos. Por otra parte, el alumno ha dejado de ser un simple receptor pasivo de los conocimientos transmitidos por el profesor, siendo su participación mucho más activa. Ya no tenemos el modelo Magister docet/Discipulo audit sino que ambos roles interactuan haciendo que la clase sea más participativa y dinámica. De este modo, y recogiendo palabras de Casado y García Bermejo, "se contempla el proceso de enseñanza-aprendizaje como algo creativo que permite al alumno explorar en el lenguaje y organizar sus nuevos conocimientos utilizando para ello sus propios esquemas organizativos" (2000: 79).

En resumen, los modelos que se siguen en la actualidad en el ámbito de la enseñanzaaprendizaje de lenguas extranjeras son aquellos que fomentan la autonomía del discente.

\title{
2.1.5. Método contrastivo
}

A mediados de los años cuarenta del siglo XX surgió el método contrastivo en el ámbito del estructuralismo de signo conductista, con la finalidad de resolver los problemas generados por el uso del lenguaje en una comunidad lingüística y, en particular, los relacionados con la enseñanza-aprendizaje de lenguas. Más específicamente, la lingüística contrastiva enfoca los efectos producidos por las diferencias entre las estructuras de la L1 y las de la L2 en el transcurso del aprendizaje, basándose en la comparación sistemática de las dos lenguas.

El primer modelo que surgió en el ámbito de la Lingüística contrastiva, fue el Análisis contrastivo. Inaugurado en 1945, alcanza su auge en 1957 con Linguistics across Cultures de Robert Lado (1968). El objetivo de este trabajo era identificar las áreas problemáticas en el proceso de aprendizaje de una segunda lengua y, en concreto, los elementos de la lengua 
materna del alumno que, por interferencia, dificultan el proceso de aprendizaje y son fuente directa de error. El transfer lleva a una fuerte tendencia a la contaminación (interferencia) que puede radicarse con la consiguiente fosilización de los errores en fases sucesivas del aprendizaje. Estos errores se pueden producir en todos los niveles de la lengua: fonológico, ortográfico, morfológico, sintáctico y léxico. Con el fin de que estos errores, debidos a interferencia, no lleguen a cristalizarse en el interlengua del alumno, es oportuno impulsar «un'analisi dinamica contrastiva», tal y como sugiere Maria Vittoria Calvi (2004: 12) y como venimos diciendo, por lo que se solicita al alumno que construya hipótesis sobre la L2 a partir de la L1. Ello lleva al desarrollo y afianzamiento de una consciencia interlingüística del alumno, a la vez que se le acostumbra a la comparación continua entre ambos idiomas, promocionando así su aprendizaje autónomo, además de ayudarle a construir un mecanismo de control que vigile sus realizaciones lingüísticas (monitor).

Sin lugar a duda, la implantación del Marco Común Europeo de Referencia de las Lenguas $^{8}$ y la difusión de certificaciones internacionales plantean más bien una didáctica desde la propia lengua; pero la creciente necesidad de mediación entre lenguas y culturas diferentes subrayada por el mismo texto europeo: "Las actividades de mediación de tipo linguiístico, que (re)procesan un texto existente, ocupan un lugar importante en el funcionamiento lingüístico normal de nuestras sociedades" (Common European Framework for Languages 2002: 2), hace aconsejable la adopción de un enfoque contrastivo, que potencie la conciencia linguística del alumno, más aun, cuando las lenguas en cuestión son lenguas afines como lo son el italiano y el español ${ }^{9}$.

\section{Conclusiones}

La finalidad de la enseñanza de lenguas extranjeras es que los alumnos sean capaces de comunicarse de manera natural en contextos reales. Se trata por lo tanto de enseñar a los estudiantes a convertirse en hablantes ilimitados. Desde el latín como lengua culta, a las actuales lenguas modernas, el método y los procedimientos para llevarlo a cabo han ido evolucionando. La idea del método idóneo ha quedado atrás por utópica e inalcanzable, aunque la visión futurista de la enseñanza de lenguas se mueve, cada vez más, hacía las funciones comunicativas de la lengua dejando atrás la gramática: en lugar de enseñar elementos gramaticales se les pedirá a los estudiantes que resuelvan situaciones problemáticas utilizando la lengua. La enseñanza se centra en el alumno y en sus necesidades, y debe promover una comunicación real, en situaciones reales.

El enfoque comunicativo, que tiene su origen en los primeros años de la década de los setenta del siglo pasado, entiende el aprendizaje de lenguas como un proceso donde lo más sustancial no son las formas lingüísticas, sino las intenciones comunicativas (el aspecto funcional) y su propiedad. No obstante, y la experiencia adquirida me respalda, partiendo del hecho de que no existe la perfección metodológica, sino un saber hacer y adaptación constante de métodos, teorías, enfoques y estilos de enseñanza-aprendizaje, y partiendo de los presupuestos metodológicos actuales, me inclino a presentar el enfoque comunicativo unido al método contrastivo, sobre todo en el estudio de lenguas afines, como el marco metodológico ideal de actuación docente, al entender que el objetivo educativo del profesor de lengua extranjera es que sus alumnos alcancen una competencia comunicativa y gramatical plenas. 


\section{Notas}

1. En su Some Thoughts Concerning Education, Locke aboga por una didáctica de las lenguas extranjeras de forma similar a la seguida en el aprendizaje de la lengua materna, esto es, mediante la imitación de modelos y la realización de ejercicios lingüísticos, y con ello se opone al conservador modelo de enseñanza-aprendizaje seguido por la Iglesia.

2. El primer autor que aboga por una transformación de los métodos empleados para la enseñanza de segundas lenguas -que son los mismos utilizados para la didáctica de las lenguas clásicas- es Viëtor, quien en 1882, en su Quosque Tandem - Der Sprachunterricht muß umkehren, denuncia la ineficacia de la metodología empleada hasta el momento.

3. Ya en 1941 la Fundación Rockeffeller había subvencionado el denominado Intensive Language Program, dada la necesidad de formar en idiomas a los soldados destinados en las Islas del Pacífico.

4. Son destacables en este sentido, dentro del ámbito de la enseñanza de la lengua francesa, los resultados obtenidos en el Ecole National Superieur de St. Cloud, dirigido por G. Labat yJ. Guénot; las investigaciones realizadas en el Centre d' etude du français élémentaire (CREDIF) o las del Centre de recherche et d' etude pour la diffousion du français; y, finalmente, las llevadas a cabo en el Bureau d' etude et de liason pour l'enseignement du français dans le monde, denominado a partir de 1965 Bureau por l'enseignement de la lange et de la civilisation française á l'entranger.

5. En el caso concreto del italiano para hispanohablantes véase el estudio de G. Marangon, del año 2007, "El video como médium didáctico para el profesor de L2. Estudio lingüístico contrastivo (italiano-español) y aplicación práctica para la clase de lengua italiana”. Analecta Malacitana AnMal electrónica. 22.

6. En la última década del pasado siglo son muchos los estudios centrados en el uso de internet y las nuevas tecnologías multimedias en la enseñanza de lenguas extranjeras. Véase a este respecto los realizados por M. Hahn, S. Künzel y G. Wazel, Multimedia - eine neue Herausforderung für den Fremdsprachenunterricht (1996), en el que los autores no sólo analizan el empleo de los medios informáticos en la enseñanza de una lengua extranjera, sino que al mismo tiempo también aportan las utilidades que dichos medios pueden ofrecer tanto al docente como al discente; R. Donath, E-MailProjekte im Englischunterricht (1996), que refleja las posibilidades que ofrece el correo electrónico para la enseñanza del inglés como lengua extranjera desde una perspectiva didáctica.

7. Podemos citar como ejemplos, no sólo en materia de enseñanza de lenguas extranjeras, sino también en otros ámbitos científicos, el presentado por Carmiña, Cuenca, Ballester y Montes, Diseño multimedia de material docente para una asignatura de control (Universidad Politécnica de Valencia), o el presentado por Gómez, Orozco, Aranda, Aplicación de un sistema generador de cursos para la enseñanza de la Automática a través de Internet (UE-CEES, Universidad Complutense, UNED).

8. En el año 2001, coincidiendo con la celebración del Año Europeo de las Lenguas, el Consejo de Europa publicó el MCERL, documento que, en su edición original en inglés, fue publicado por la Universidad de Cambridge con el título Common European Framework of Reference for Languages: Learning, teaching, assesment. En el año 2010 las Áreas de lengua italiana, inglesa, alemana y francesa del Departamento de Traducción e Interpretación, Lenguas Romances, Estudios Semíticos y Documentación de la Universidad de Córdoba, elaboraron un Proyecto de mejora docente cuya finalidad es la de ofrecer una página web de fácil consulta para la comunidad universitaria en la que se pueda encontrar información detallada sobre los objetivos del Marco común de las lenguas y los principales agentes certificadores para los niveles de competencia lingüística. El proyecto se titula "Evaluación de las competencias lingüísticas para una lengua extranjera según la normativa del marco común europeo de referencia (MCER)". http://aulavirtual.uco.es/presentacion/pruebas/mcer/?dst=objetivos.

9. G. Marangon. 2009. "Italiano y español: lenguas afines. Importancia de la L1 en el proceso de aprendizaje de la L2". Analecta Malacitana, AnMal electrónica. 27: 185-194; 2011. "Estudio contrastivo del léxico en las lenguas afines. La engañosa semejanza: la traducción de los falsos amigos en español e italiano", Analecta Malacitana, AnMal electrónica. 30: 285-292; 2012. La importancia de la gramática contrastiva en el ámbito traductológico de las lenguas afines: el caso concreto del italiano y del español, Granada: Editori. 


\section{Bibliografía}

Breindl, E. 1987. Präpositionalobjekte und Präpositionalobjektsätze im Deutschen. Tübingen: Niemeyer.

Blanco, Carmen F. 2010. "Evaluación de las competencias lingüísticas para una lengua extranjera según la normativa del marco común europeo de referencia (MCER)". Recuperado de: http://aulavirtual.uco.es/presentacion/pruebas/mcer/?dst=objetivos Consulta: 13 de febrero de 2012.

Calvi, M.V. 2004. “Aprendizaje de lenguas afines: español e italiano”. RedELE. 1: 1-22.

Camiña, C., A. Cuenca, E. Ballester y A. Montes. 2001. "Diseño multimedia de material docente para una asignatura de control". En: Acta de las II Jornadas de Trabajo "Enseñanza via Internet/Web de la Ingeniería de Sistemas y Automática” EIWISA’01. Actas de las Jornadas. UNED.

Casado Casado J. y M. L. García Bermejo. 2000. “Consideraciones didácticas sobre la enseñanza de lenguas extranjeras asistidas por ordenador”. Didáctica. Lengua y Literatura. 12: 67-89.

Common european framework for languages: learning, teaching, assessment. 2002. Strasbourg: Instituto Cervantes para la Traducción en Español.

Donath, R. 1996. E-Mail-Projekte im Englischunterrcht. Stuttgart: Klett.

Gómez, E., J. López y B. Aranda. 2001. “Aplicación de un sistema generador de cursos para la enseñanza Autónoma a través de internet". IFAC. 1-7.

Hahn, M., S. Künzel y G. Wazel. 1998. Multimedia-eine neune Herausforderung für den Fremdsprachenunterricht. Frankfurt: Peter Lang.

Lado, R. 1968. Linguistic across Cultures: Applied Linguistics for Language Teachers. Michigam: Ann Arbor.

Locke, J. 1963. Some thoughts concerning human education. London.

Marangon, G. 2007. “El video como médium didáctico para el profesor de L2. Estudio lingüístico contrastivo (italiano-español) y aplicación práctica para la clase de lengua italiana”. Analecta Malacitana, AnMal electrónica. 22: 1-15.

2009. "Italiano y español: lenguas afines. Importancia de la L1 en el proceso de aprendizaje de la L2”. Analecta Malacitana, AnMal electrónica. 27: 185-194.

2011a. "Estudio contrastivo del léxico en las lenguas afines. La engañosa semejanza: la traducción de los falsos amigos en español e italiano". Analecta Malacitana, AnMal electrónica. 30: 285-292.

2011b. La importancia de la gramática contrastiva en el ámbito traductológico de las lenguas afines: el caso concreto del italiano y del español. Granada: Educatori.

Richards, J. y R. Schmidt. 1997. Longman Dictionary of Language Teaching and Applied Linguistics. $7^{\text {th }}$ Edition. Malysia: Longman.

Ruipérez, G. 1997. “La enseñanza de lenguas asistida por ordenador (ELAO)”. Carabela. 42: $5-25$. 
Sass, A. 2007. "Filme im Unterricht-Sehen(d) lernen”. Fremdsprache Deutsch. 36: 5-13.

Schlickau, S. 2000. Neune Medien in der Sprach-und Kulturvermittlung. German Edition.

Storch, N. 1997. “The editing talk of adult learners”. Language Awareness. 6 (4): 221-232.

1998. “Comparing second language learners' attention to form across tasks”. Language Awareness. 7 (4): 176-191.

Vaughan, T. 2008. Si quieres, puedes. Madrid: Libros-libres.

Viëtor, W. 1882. Der Sprachunterrcht muß umkehrenl Ein Beitrag zur Überßürdungsfrage von Quosque Tandem. Heilbronn: Gerbr. Henninger. 